\section{PWE-102 CAPSULE ENDOSCOPY IN SUSPECTED SMALL BOWEL CROHN'S DISEASE-IS IT WORTH REPEATING A NEGATIVE STUDY?}

${ }^{1}$ Alexander Robertson*, ${ }^{1}$ Diana Yung, ${ }^{2}$ Drlan Arnott, ${ }^{1}$ Anastasios Koulaouzidis. ${ }^{1}$ Department of Gastroenterology, Royal Infirmary of Edinburgh, Edinburgh, UK; ${ }^{2}$ Department of Gastroenterology, Western General Hospital, Edinburgh, UK

\subsection{6/gutjnl-2018-BSGAbstracts.336}

Introduction Crohn's disease (CD) affects the small bowel (SB) ${ }^{1}$ in a significant proportion of patients. Capsule endoscopy (CE) has a high diagnostic yield (DY) for SB CD and inflammation ${ }^{2}$; however, in patients with negative initial CE but ongoing clinical suspicion of $\mathrm{CD}$, information on the utility of repeat $\mathrm{CE}$ is limited.

\begin{tabular}{|c|c|c|c|c|c|c|}
\hline Pt & $\begin{array}{l}\mathrm{M} / \\
\mathrm{F}\end{array}$ & $\begin{array}{l}\text { Age } \\
\text { (yrs) }\end{array}$ & Initial CE findings & $\begin{array}{l}\text { Repeat CE } \\
\text { findings }\end{array}$ & $\begin{array}{l}\text { Time } \\
\text { between CEs } \\
\text { (days) }\end{array}$ & FC results \\
\hline 1 & $\mathrm{~F}$ & 44 & $C D$ & $\begin{array}{l}\text { Consistent with CD } \\
\text { diagnosis }\end{array}$ & 48 & High \\
\hline 2 & M & 16 & $C D$ & Active SB CD & 862 & High \\
\hline 3 & $\mathrm{~F}$ & 40 & Likely CD & Normal & 544 & High \\
\hline 4 & $\mathrm{~F}$ & 24 & Likely CD & Nonspecific & 454 & $\begin{array}{l}\text { High }(x 2) \\
\text { normal }(x 5)\end{array}$ \\
\hline 5 & $\mathrm{~F}$ & 66 & $\begin{array}{l}\text { Likely } C D \text {, ileal } \\
\text { retention }\end{array}$ & $\begin{array}{l}\text { Ileal strictures and } \\
\text { bleeding; retention }\end{array}$ & 2206 & High \\
\hline 6 & M & 30 & $\begin{array}{l}\text { Nonspecific } \\
\text { inflammation }\end{array}$ & Normal & 2269 & High \\
\hline 7 & $\mathrm{~F}$ & 15 & $\begin{array}{l}\text { Nonspecific } \\
\text { inflammation }\end{array}$ & Normal & 285 & Normal \\
\hline 8 & $\mathrm{~F}$ & 57 & $\begin{array}{l}\text { Nonspecific } \\
\text { inflammation }\end{array}$ & $\begin{array}{l}\text { Nonspecific } \\
\text { inflammation }\end{array}$ & 659 & High \\
\hline 9 & M & 53 & Nonspecific ileitis & Normal & 1560 & High \\
\hline 10 & $\mathrm{~F}$ & 23 & $\begin{array}{l}\text { Nonspecific } \\
\text { findings }\end{array}$ & Suggestive of CD & 2066 & High \\
\hline 11 & $\mathrm{~F}$ & 54 & $\begin{array}{l}\text { Nonspecific } \\
\text { findings }\end{array}$ & Suggestive of $C D$ & 421 & High \\
\hline 12 & $\mathrm{~F}$ & 64 & $\begin{array}{l}\text { ? } \\
\text { lymphoproliferative } \\
\text { disorder }\end{array}$ & $\begin{array}{l}\text { ?inflammatory/ } \\
\text { infiltrative process }\end{array}$ & 377 & High \\
\hline 13 & M & 44 & NSAID enteropathy & $\begin{array}{l}\text { Likely NSAID- } \\
\text { related ulcer }\end{array}$ & 1063 & Normal \\
\hline 14 & $\mathrm{~F}$ & 61 & Angioectasia & $\begin{array}{l}\text { Duodenal } \\
\text { angioectasias }\end{array}$ & 538 & High \\
\hline 15 & $\mathrm{~F}$ & 36 & Duodenal ulcer & Normal & 3123 & High \\
\hline 16 & $\mathrm{~F}$ & 28 & Normal & Normal & 183 & High \\
\hline 17 & $\mathrm{~F}$ & 29 & Normal & Normal & 91 & $\begin{array}{l}\operatorname{High}(x 1) \\
\text { normal }(x 2)\end{array}$ \\
\hline 18 & M & 65 & Stomach retention & Normal & 70 & $\begin{array}{l}\text { None } \\
\text { available }\end{array}$ \\
\hline 19 & $\mathrm{~F}$ & 64 & Recorder failure & Nonspecific ileitis & 920 & High \\
\hline
\end{tabular}

Methods Using a prospectively-maintained database, we identified patients undergoing repeat $\mathrm{CE}$ for suspected SB CD at a tertiary care centre. Over the data collection period (20052017), CEs were reported based on clinical impression without use of a standardised tool (i. e. Lewis score or CECDAI).
Results Over the study period, 434 CEs were carried out for suspected SB CD. 19 (4.4\%) were repeat CEs, median age of patients 44.3 years (range 15.8-66.8), 14F/5M. The median time between CEs was 544 days (range 48-3123).

Conclusion The DY of repeat CE carried out for suspected SB $\mathrm{CD}$ was 6/17 (35.3\%) where there had been diagnostic uncertainty following the initial CE. In patients where no SB inflammation was seen on the initial CE, $0 / 5(0 \%)$ of repeat CEs showed inflammatory changes.

Although the numbers in this cohort study are small, our findings would support the hypothesis that repeat CE is useful in equivocal and inconclusive studies where there is clinical suspicion of SB CD. Conversely, in patients whose initial CE showed no evidence or suggestion of SB CD, repeating the procedure adds little.

\section{PWE-103 CAPSULE ENDOSCOPY IN SUSPECTED GI BLEEDING-IS IT WORTH REPEATING A NEGATIVE STUDY?}

Alexander Robertson*, Diana Yung, Sarah Douglas, Chris Fraser, Anastasios Koulaouzidis John,. Royal Infirmary of Edinburgh, Edinburgh, UK

\subsection{6/gutjnl-2018-BSGAbstracts.337}

Introduction The most common indications for capsule endoscopy (CE) are iron deficiency anaemia (IDA) and occult gastrointestinal bleeding (OGIB). This study aimed to assess the diagnostic yield (DY) of repeat CE in patients for whom there is ongoing clinical concern, despite an initially negative CE.

Methods Patients who underwent $\geq 2$ CEs for IDA/OGIB at our centre, from 2005 to 2017, were identified from a prospectively-designed database. Data were extracted on indications and CE findings. The capsule examination was considered to have DY if the findings accounted for the patient's presentation.

Results 85 patients underwent repeat CE during the study period, median age 65.8 years (range $11.5-89.8 ; 42 \mathrm{~F} / 55 \mathrm{M}$ ). The median interval between procedures was 463 days (range 1-3066). 14 patients underwent repeat CE due to a retained or incomplete initial capsule and were excluded from analysis. In the remaining 71 patients, initial CE findings were: normal (22), vascular lesions/bleeding (26), small bowel inflammation (5), others (8; including polyps, portal hypertensive enteropathy (PHE), celiac disease, small bowel (SB) varices and SB lymphoma), nonspecific findings of unclear significance (6) and non-SB findings (5).

In patients with a normal initial $\mathrm{CE}$, repeat $\mathrm{CE}$ identified a cause for IDA/OGIB in 9/22 (40.9\%). 12/22 CEs (54.5\%) were normal and 1 was incomplete.

Of the 19 patients with vascular lesions seen initially, the initial lesion was confirmed in 13/19 (68.4\%) CEs. The diagnosis was revised in 2 patients: 1 was found to have PHE and 1 likely NSAID enteropathy. 3/19 (15.8\%) patients had normal repeat CEs and 1 was retained.

7 patients had active bleeding on initial CE but no lesion seen. Repeat CE in this group had DY 5/7 (71.4\%): angioectasias (3), polyp (1), SB inflammation (1). 2 repeat CEs were normal.

In the 6 patients with nonspecific initial findings, repeat CE identified specific findings in $2 / 6$ (33.3\%) patients (1 NSAID enteropathy, 1 jejunal ulcer). 
10 patients underwent a 3rd CE. In 7/10 patients with concordant initial CEs, the DY of repeat CE was 0/7. Where the 2 initial CEs disagreed, DY was 2/3.

Conclusion 1. In patients with a negative or inconclusive initial CE for IDA or OGIB, repeating the procedure has an overall DY of $25 \%(7 / 28)$.

The DY is highest when fresh blood was seen in the initial procedure $(71.4 \%)$ even if no lesions were found initially.

Patients with initially normal studies had lower DY (22.7\%).

3rd CE is only warranted by a change in presentation or discordance in the previous results, especially when one examination has identified active bleeding.

\section{PWE-104 IDA PATIENTS WITH NEGATIVE COELIAC SEROLOGY ON STRAIGHT TO TEST PATHWAY; IS D2 BIOPSY NECESSARY?}

${ }^{1}$ Faisal Shaikh*, ${ }^{1}$ Peter Mooney, ${ }^{2}$ Jason Jennings. 'Leeds Teaching Hospitals NHS Trust, Leeds, UK; ${ }^{2}$ Leeds Teaching Hospitals NHS Trust, Leeds, UK

\subsection{6/gutjnl-2018-BSGAbstracts.338}

Introduction Current BSG guidelines for Iron Deficiency Anaemia (IDA) recommend screening all adult patients for coeliac disease (CD). Duodenal biopsies are only required in patients with positive coeliac serology due to very low post-test probability for CD if serology is negative. BSG CD guidelines however recommend duodenal biopsies regardless of the serology result. We aimed to assess current practise and outcomes in the setting of a straight to test (STT) IDA pathway.

Methods We conducted a retrospective analysis of all adult patients referred on STT IDA pathway over a 3 months period. Patients who did not ultimately undergo endoscopy or had a prior diagnosis of $\mathrm{CD}$ were excluded from the study.

Results During the study period 239 patients were referred under the STT IDA pathway. Of these, 175 (male 76, female 99) underwent endoscopic investigations and were included in the study. Mean age of male and female participants was 66 and 68 years respectively. The average haemoglobin on referral was $102 \mathrm{~g} / \mathrm{L}$. Pre-endoscopy coeliac serology was only available in $44 / 175$ patients $(25.1 \%)$. Serology was positive in 1 of these patients (2.3\%)-CD was confirmed on duodenal biopsy. Duodenal biopsies were still taken in 31/43 (72.1\%) patients with negative serology, histology was normal in all cases. $110 / 131(84 \%)$ of patients without pre-endoscopy serology had duodenal biopsies taken. 9/110 (8.2\%) had abnormal duodenal biopsies. 4 cases intraepithelial lymphocytosis, 2 duodenitis and 1 Giardiasis. 2 patients had villous atrophy with suspected CD-serology came back positive in 1 patient. Second patient awaiting further investigations. There was no difference in duodenal biopsy rate based on $\mathrm{CD}$ serology availability ( $72 \%$ vs $84 \% \mathrm{p}=0.11$ ).

Conclusions Patients in STT IDA pathway with negative CD serology are unlikely to have CD. Duodenal histology is abnormal in a significant number of patients with negative serology however failure to check CD serology prior to endoscopy leads to diagnostic uncertainty and delays in diagnosis. A point of care test for CD performed in endoscopy could fill this gap. Incongruent anaemia and $\mathrm{CD}$ guidelines lead to uncertainty amongst clinicians and may explain variable practise.
PWE-105 IS THERE A CORRELATION BETWEEN SEVERITY OF BILE ACID MALABSORPTION (BAM) AND RESPONSE TO TREATMENT?

${ }^{1} \mathrm{~W}$ Siu*, ${ }^{1} \mathrm{~K}$ Ko, ${ }^{2} \mathrm{~F}$ McKiddle, ${ }^{1} \mathrm{~F}$ Clegg, ${ }^{1} \mathrm{G}$ Bain, ${ }^{1} \mathrm{~A}$ McKinlay. ${ }^{1}$ Department of Digestive Disorders, Aberdeen Royal Infirmary, Aberdeen, UK; ${ }^{2}$ Nuclear Department, Aberdeen Royal Infirmary, Aberdeen, UK

\subsection{6/gutjnl-2018-BSGAbstracts.339}

Introduction NICE guidelines in 2012 have recommended SeHCAT to be used in research in order to collect more information in its usefulness in the diagnosis and treatment of BAM. A previous study has identified a lack of consistent cutoff threshold values for abnormal SeHCAT results. The aim of this study is to determine whether there is any relationship between the severity of BAM and treatment response.

Methods Medical records of 492 patients who had a SeHCAT scan at Aberdeen Royal Infirmary between 23/7/2013 to 9/6/ 2017 were retrospectively reviewed. Mild, moderate and severe BAM were defined as $10.1 \%-15 \%, \quad 5.1 \%-10 \%$ and $<5 \%$ retention of SeHCAT after one week respectively. Data including the severity and types of BAM were recorded. Treatment responses to bile acid binders were also recorded when patients were followed up in the clinic after the scans.

Results 492 scans were performed and 51\% (252/492) of patients had abnormal SeHCAT results $(<15 \%)$ over the study period. The mean age was 51.6 with a female predominance of $69 \%(174 / 252)$.

$20 \%(50 / 252)$ of these patients had a prior diagnosis of IBS and 27\% (67/252) patients had previous cholecystectomy.

$17 \%$ (44/252) had type 1 BAM, 53\% (134/252) had type 2 BAM and 29\% (74/252) had type 3 BAM. The mean SeHCAT retention percentage was $2.59 \%$ for type 1 BAM, $7.45 \%$ for type 2 BAM and $5.63 \%$ for type 3 BAM. The difference was statistically significant $(\mathrm{p}<0.001)$.

$52 \%(132 / 252)$ of patients had treatment response documented following their scans and $13 \%$ (17/132) of these patients stopped treatment due to side effects.

For the remaining 115 patients, 71\% (12/17) of patients with mild BAM had good response to bile acid binder compared to 77\% (23/30) with moderate BAM and 78\% (55/68) with severe BAM. The difference was not statistically significant $(\mathrm{p}<0.635)$.

15 out of 90 patients who responded to colesevelam previously found cholestyramine ineffective or intolerance of it. Conclusions In our study, the mean SeHCAT retention level was significantly lower for BAM type 1 compared to BAM types 2 and 3 . There was an overall good therapeutic response to bile acid binders in patients with a positive $\mathrm{SeH}$ CAT scan. However, there was no statistically significant difference between severity of BAM and theurapeutic response.

Further prospective study using larger sample size is required to assess the accuracy and cut-offs of the SeHCAT test in diagnosing BAM as determined by theurapeutic response to BAS treatment.

\section{PWE-106 CHANGES IN THE TESTING FOR AND INCIDENCE OF COELIAC DISEASE IN THE UK 2005-2015}

${ }^{1,2}$ Joe West ${ }^{*},{ }^{1,3}$ Harmony Otete, ${ }^{4}$ Alyshah Sultan, ${ }^{1,2,5}$ Colin Crooks. ${ }^{1}$ Division of Epidemiology and Public Health, University Of Nottingham, Nottingham, UK; ${ }^{2}$ NIHR Nottingham Biomedical Research Centre, Nottingham, UK; ${ }^{3}$ School of Pharmacy, University of Nottingham, Nottingham, UK; ${ }^{4}$ Research Institute for Primary Care and Health Sciences, Keele University, Keele, UK; ${ }^{5}$ Nottingham Digestive Diseases Centre, Nottingham, UK

10.1136/gutjnl-2018-BSGAbstracts.340 